CLAWAR 2018: 21st International Conference on Climbing and Walking Robots and the Support Technologies for Mobile Machines, Panama City, Panama, 10-12 September 2018

\title{
ROBOTICS FOR FACIAL RECONSTRUCTION USING MEDICAL IMAGES
}

\author{
JOSÉ SERRACÍN, IVETH MORENO, RONY CABALLERO \\ Faculty of Electrical Engineering, Universidad Tecnológica de Panamá, Chiriqui, \\ Panama \\ HECTOR MONTES \\ Faculty of Electrical Engineering, Universidad Tecnológica de Panamá, Panama; \\ Robotics of Exteriors and Services Group, Center of Automatics and Robotics, CSIC- \\ $U P M$, Spain
}

\begin{abstract}
The presented study in this paper deals with the development of a cranio-maxillofacial reconstruction system in three dimensions, which will interact with a haptic tool. In turn, a specific robot will be controlled using this tool, which it has installed applications associated with surgery. The proposed system will help physicians gain pre-operative experience, based on reconstructions of three-dimensional images taken from real patients. Good results have been obtained, which validate the proposed system.
\end{abstract}

\section{Introduction}

This article deals with the topic of cranio-maxillofacial reconstruction using medical images in two dimensions (2D) for its reconstruction in three dimensions (3D) within a virtual scenario, and the integration of a tele-operated robot by means of a haptic joystick. Specifically, it will refer to tests performed with the robot in bone machining tasks. In addition, in this work, the results obtained on the study and validation of the tele-operated system to perform simulated craniomaxillofacial surgeries are presented.

The main objective of using a robot in maxillofacial reconstruction is to propose the use of a system that allows the doctor to obtain hours of effective preoperative practices for surgeries.

The article is structured as follows: in Section 1, introduction. Section 2, a brief state-of-the-art has been introduced. In Section 3, the description of the system will be presented. Section4, presents some experimental results. Finally, conclusions and future work are presented in Section 5. 


\section{Background}

Among the most presented cases in the emergency rooms of hospitals in Latin America and worldwide, are the traumatic brain injuries (TBI), which are any structural or functional injury of the skull and / or its secondary content due to an abrupt exchange of energy mechanical [1]. This is a problem with a high incidence of mortality and morbidity in patients of productive age, affecting mainly the population between 15 to 45 years [2]. The main and most common cause of this problem is traffic accidents, with a $75 \%$, approximately. A large percentage of survivors of this situation will have disabling sequelae [3], if they have not an early diagnosis and an adequate therapeutic strategy.

In order to help in this type of problem, training programs have been implemented to perform laparoscopic procedures, thus acquiring a fundamental role in surgical training. This training is intended for either resident doctors or surgeons, who wish to practice in new minimally invasive techniques, previously to surgery in patients [4]. In these surgical procedures, it has been required to change the model of surgical teaching that they had for more than a century of use, giving rise to the development and application of new simulation techniques, and bringing with them a tutored and optimized teaching, with more dexterity and fewer mistakes. In this way, better skills of the trained subject have been developed and the risk of subjecting patients to surgical errors and their potential subsequent damage is diminished, compared with the classic teaching model [5].

The presented study deals with the development of a cranio-maxillofacial reconstruction system in three dimensions, which will interact with a haptic tool that will also control a robot, with applications associated with surgery, also allowing tests with pig bones. The proposed system will allow the surgeon to perform preoperative tests in a simulated environment, with images of real patients. It should be noted that the surgeon can acquire some certain skills through the use of the robot to make more precise and reliable cuts and/or perforations.

\section{Description of the system}

The system that was developed consists of a user interface, which has an application which is fed by a stack of real cranio-maxillofacial images in two dimensions (2D), for reconstruction to a virtual solid in three dimensions (3D). The user interface was developed from the QT4 libraries, which is a crossplatform application development kit. The interface has a user-friendly environment. Through it, imagery files can be imported and, besides, they can be configured with the imagery acquired parameters. 
The images are reconstructed generating a solid in three dimensions (3D), using the VTK libraries and visualized in a graphic environment within the interface created with QT4. At this point, the virtual representation of the proxy (final effector) of the Phantom Omni haptic joystick is added to the rendered image. The haptic joystick allows having a force feedback, which helps the operator in the control of the end-effector.

The joystick is in charge of controlling the end-effector of the robot that will perform the machining tasks in the bone, such as drilling, roughing, etc. In addition, the joystick will give the surgeon a feeling that he is in a real environment, guaranteeing the possibilities of a better performance during the surgery.

The robot used in this system was designed and assembled exclusively for this purpose. The robot is a two-bar parallel type, with two modular degrees of freedom [6], and with all the characteristics of a robot with a parallel structure: speed, precision, and small workspace, among others.

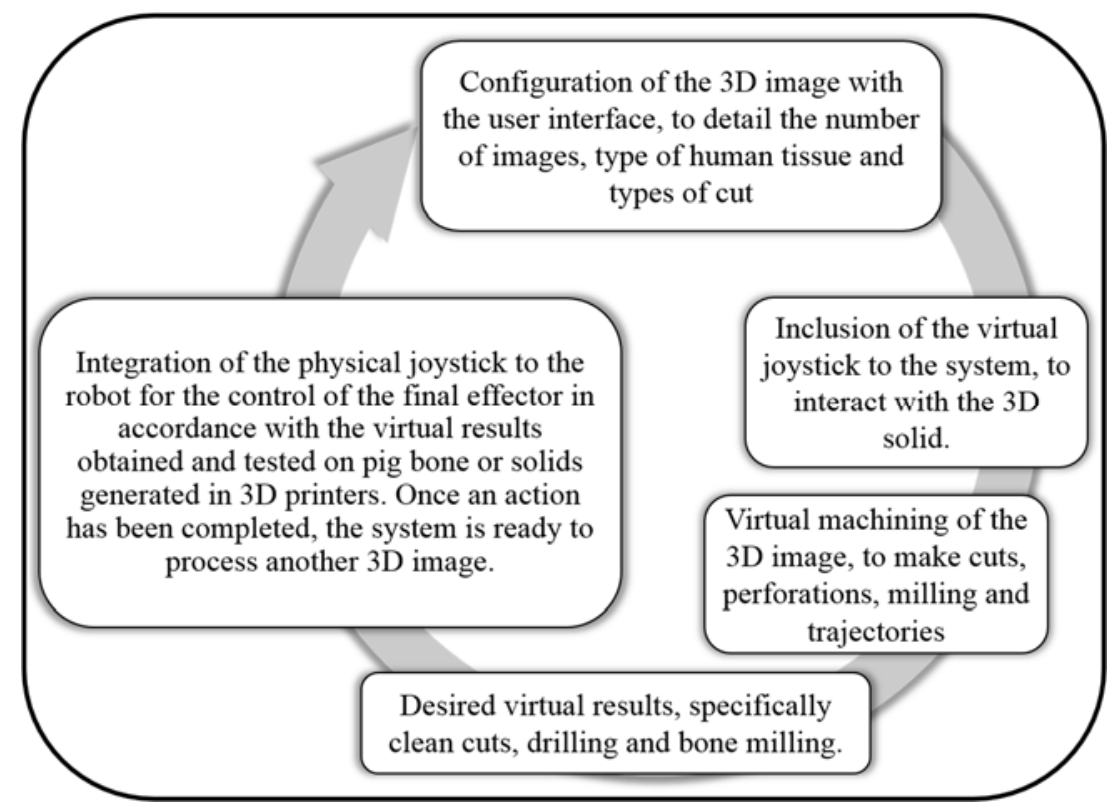

Figure 1. Description of the functioning of the facial reconstruction system using robotics.

The operation of the system can be considered in two phases: a first phase in which all virtual tests will be performed with the 3D reconstruction of skull- 
maxillofacial images and the haptic joystick, until obtaining the desired results; and a second phase in which the joystick controls the end effector of the robot to perform tests on solids such as skulls printed with 3D printers or on the bones of animals such as pigs. The description of the system is summarized in Figure 1, and the interaction between each of the elements that comprise it is shown in Figure 2 .

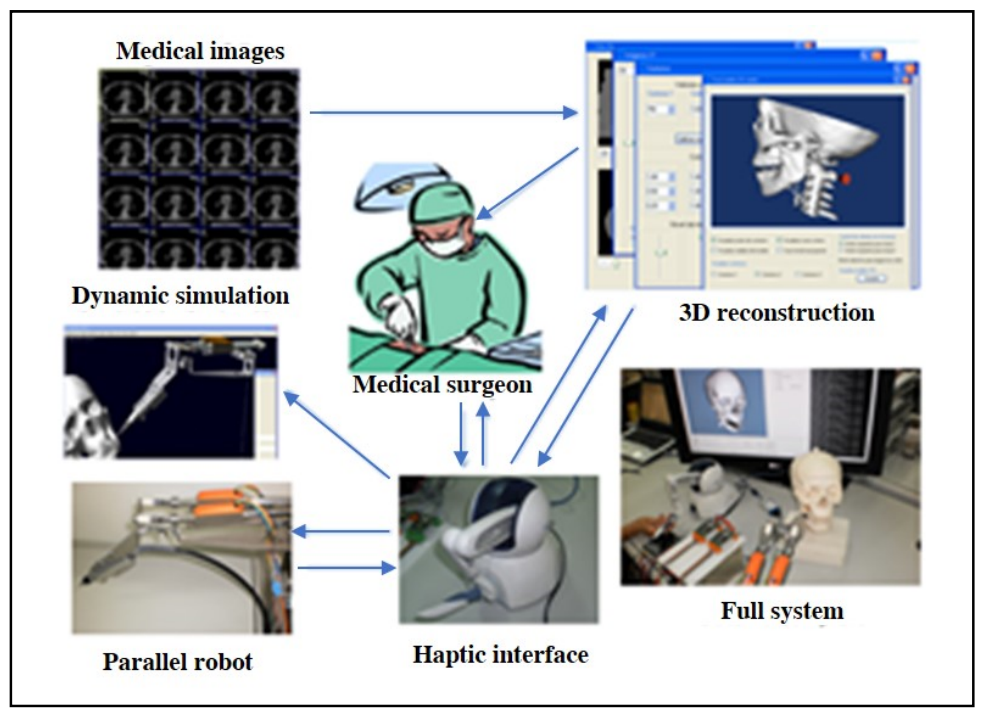

Figure 2. Interaction between the elements that make up the complete system.

\section{Experimental results}

The experimental tests of the proposed system were carried out with pig bones (see Figure 3), since this type of bone is very similar to the bone tissues of the human being, but stronger. To perform the machining tests on the bones, a drill bit placed in the end effector of the robot is approached at a constant speed of 8 millimeters per second ( $\mathrm{mm} / \mathrm{s})$, approximately. Once the bone is reached and it is touched with the drill, the approach speed decreases to $0.25 \mathrm{~mm} / \mathrm{s}$, so that when the bone is drilled, no pressure is exerted that could splinter or break the bone during the drilling task. 


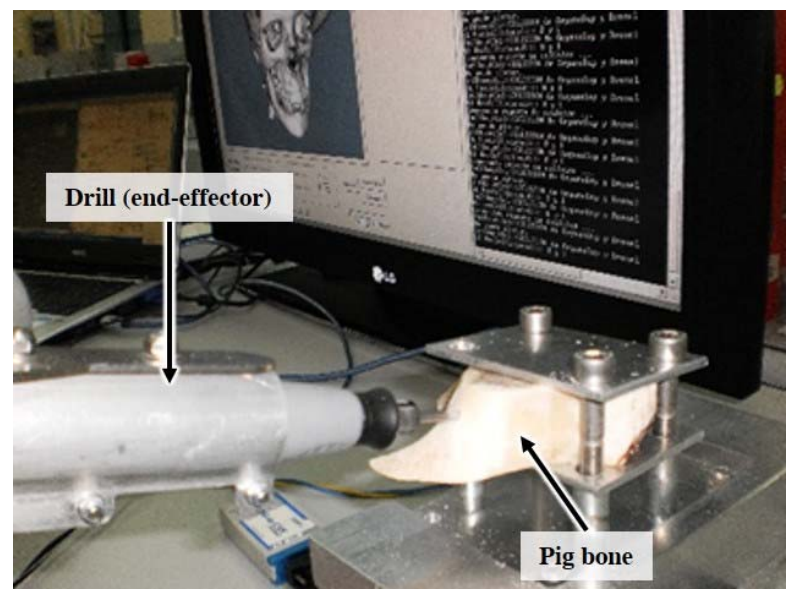

Figure 3. Final effector drilling a pig bone

The force exerted by the end effector on the bone is controlled taking into account the feedback obtained from the values of working current measurements made to each motor (see Figure 4). The approach speed control system is constantly being updated. So, if the currents increase above the maximum working current of the motors (around $450 \mathrm{~mA}$ ), then the approach speed will be immediately reduced until the current consumption is maintained within the stipulated ranges (see Figure 5). Preventing the bone from fracturing or splintering.

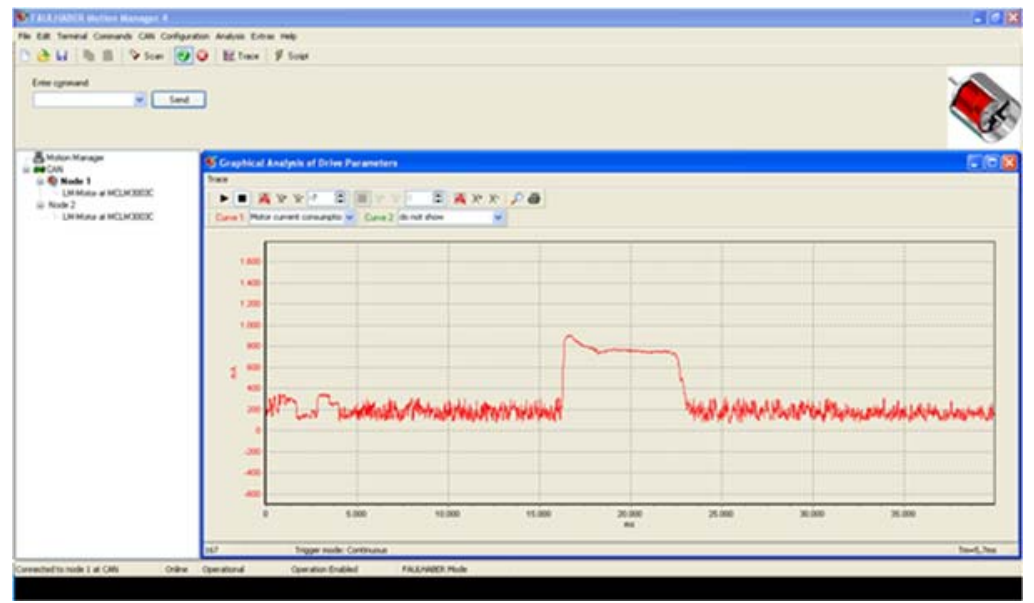

Figure 4. Measurement of the working current in motor 1 (without speed control). 


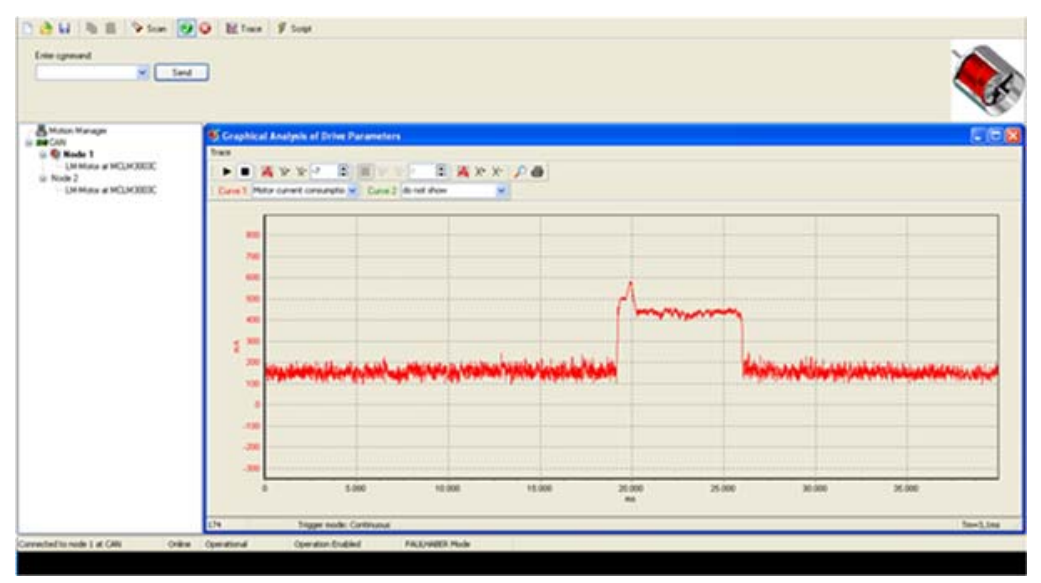

Figure 5. Measurement of the working current in motor 1 (with speed control).

The end-effector speed has been controlled by means of a PID controller. This controller has been implemented in LabVIEW, and in the user interface some values capture boxes have been placed in order to set the PID control parameters in any moment. The objective of this is to correct the approaching speed of the end-effector to the bone during the experimental tests. It is clear this manual control is only during the firsts experimental tests.

\section{Conclusions and future work}

The graphics interface presents all the options for an adequate treatment of 3D imagery, such as the movements of the solid generated in any direction, approaching and moving away from the solid, the making cuts, etc.

The full system (integrated by the robot with a parallel structure and by the haptic tool that in turn serves to control and guide the end-effector of the robot, product of the interaction of the haptic tool with the solid generated in 3D reconstruction) has given results satisfactory in terms of replication of guided trajectories, keeping consonance with the positions and orientations required.

It is expected as a product of future work the robot meets the requirements necessary to perform preoperative exercises of cranio-maxillofacial interventions. Besides, it will be created an algorithm, which automatically will generate the trajectories that the robot must perform. This will allow integrating surgical navigation with a robotic tool in the same application. 


\section{Acknowledgements}

The authors thank the support of the Universidad Tecnológica de Panamá, and the National Secretariat of Science and Technology and Innovation (SENACYT) for the development of this project. Hector Montes thanks the National System of Researchers of Panama (SNI-SENACYT) by the support to their members. Hector Montes also acknowledges support from the Centre for Automation and Robotics CSIC-UPM.

\section{References}

1. M. A. Hernández, A. J. Felipe, A. D. Mazariegos and M. L. Campos, Caracterización epidemiológica y clínica del trauma craneoencefálico producido por accidentes de tránsito, Guatemala, p. 87 (2017).

2. R. Carrillo and J. M. Meza, Trauma craneoencefálico, Revista Mexicana de Anestesiología, 38, nº 3, pp. S433-S434 (2015).

3. A. Cabrera, O. Martínez, A. Ibarra, R. Morales, G. Laguna and M. Sánchez, Traumatismo Craneoencefálico Severo, Revista de la Asociación Mexicana de Medicina Crítica y Terapia Intensiva, XXIII, no 2, pp. 94-101 (2009).

4. F. León, J. Varas, E. Buckel, F. Crovari, F. Pimentel, J. Martínez, N. Jarufe and C. Boza, Simulación en cirugía laparoscópica, Cirugía Española, 93, $\mathrm{n}^{\circ}$ 1, pp. 4-11 (2015).

5. N. Kerrigan, Simulación, ¿una necesidad en el entrenamiento para la cirugía laparoscópica colorrectal?, Revista Chilena de Cirugía, 69, nº 6, pp. 508-512 (2017).

6. J.R. Serracín, L.J. Puglisi, R. Saltaren, G. Ejarque, J.M. Sabater-Navarro and R. Aracil, Kinematic analysis of a novel 2-d.o.f. orientation device, Robotics and Autonomous Systems 60, 852-861 (2012). 